\title{
Grown-in beryllium diffusion in indium gallium arsenide: An ab initio, continuum theory and kinetic Monte Carlo study
}

\author{
Wenyuan Liu, ${ }^{1}$ Mahasin Alam Sk, ${ }^{2}$ Sergei Manzhos,,${ }^{2, *}$ Ignacio \\ Martin-Bragado, ${ }^{3, \dagger}$ Francis Benistant, ${ }^{4}$ and Siew Ann Cheong ${ }^{1}$ \\ ${ }^{1}$ Division of Physics and Applied Physics, School of Physical and Mathematical Sciences, \\ Nanyang Technological University, 21 Nanyang Link, Singapore 637371 \\ ${ }^{2}$ Department of Mechanical Engineering, National University of Singapore, Singapore 117576 \\ ${ }^{3}$ IMDEA Materials Institute, Madrid, Spain 28906 \\ ${ }^{4}$ GLOBALFOUNDRIES Singapore Pte Ltd., 60 Woodlands Industrial Park D Street 2, Singapore 738406
}

(Dated: October 1, 2018)

\begin{abstract}
A roadblock in utilizing InGaAs for scaled-down electronic devices is its anomalous dopant diffusion behavior; specifically, existing models are not able to explain available experimental data on beryllium diffusion consistently. In this paper, we propose a comprehensive model, taking selfinterstitial migration and Be interaction with Ga and In into account. Density functional theory (DFT) calculations are first used to calculate the energy parameters and charge states of possible diffusion mechanisms. Based on the DFT results, continuum modeling and kinetic Monte Carlo simulations are then performed. The model is able to reproduce experimental Be concentration profiles. Our results suggest that the Frank-Turnbull mechanism is not likely, instead, kick-out reactions are the dominant mechanism. Due to a large reaction energy difference, the Ga interstitial and the In interstitial play different roles in the kick-out reactions, contrary to what is usually assumed. The DFT calculations also suggest that the influence of As on Be diffusion may not be negligible.
\end{abstract}

\section{INTRODUCTION}

There is significant research interest in InGaAs as a promising candidate for future generation CMOS (complementary metal-oxide semiconductor) devices (specifically, for very advanced technologies e.g. $5 \mathrm{~nm}$ node and below) due to its considerably higher electron mobility compared to Silicon. The high electron mobility and a lattice constant that matches with InP make $\operatorname{In}_{0.53} \mathrm{Ga}_{0.47}$ As an ideal candidate for such devices. ${ }^{1}$ Beryllium has been considered to be an important and attractive p-type dopant due to a high activation ratio and the existence of well-developed and controllable doping methods. ${ }^{2}$ However, Be diffusion in InGaAs is extremely fast, with a diffusivity five orders of magnitude larger than in GaAs at the same temperature. ${ }^{3}$ Therefore, much effort has been devoted to investigate and understand the Be diffusion behavior, including experiments and simulations. Nevertheless, many questions are still unresolved. In particular, there is still no agreement on the mechanism and particles' charge states for Be diffusion in InGaAs. ${ }^{4-6}$

The mechanism that governs Be diffusion was widely assumed to be the kick-out mechanism $\left(\mathrm{Be}_{\mathrm{III}}+\right.$ $\left.\mathrm{I}_{\mathrm{III}} \rightleftharpoons \mathrm{Be}_{\mathrm{i}}\right)^{2,5} \mathrm{Be}_{\mathrm{III}}$ denotes a Be atom in a group-III sublattice position that is considered to be immobile while $\mathrm{Be}_{\mathrm{i}}$ is a $\mathrm{Be}$ atom in an interstitial position that has a high mobility; $\mathrm{I}_{\mathrm{III}}$ represents $\mathrm{Ga}$ and In interstitials. Usually, local thermodynamic equilibrium of diffusion process is also assumed. With this model assumption, some works were able to match experimental data. However, further clarifications about these works need to be made: (i) samples grown under similar conditions have quite different diffusion parameters in these models; (ii) although the local thermodynamic equilibrium assumption makes the model simple and tractable, it is not a very reasonable one, considering that Be diffusion is very fast; (iii) the parameters used in these models, such as the reaction energy and diffusion parameters, are not extracted from or validated by other independent self-diffusion and in-diffusion experiments or ab initio calculations, but are merely fitting parameters, making these models less predictive; and (iv) these models treated In and Ga as effectively the same kind of atom and assumed that As is not involved in Be diffusion. Such assumptions are not intuitive.

Some modified models have been proposed in order to overcome the above limitations. ${ }^{6}$ Recently, Koumetz et $a l$. proposed a combined diffusion mechanism, which removes the local thermodynamic equilibrium assumption and also takes the Frank-Turnbull (dissociative) mechanism $\left(\mathrm{Be}_{\mathrm{i}}+\mathrm{V}_{\mathrm{III}} \rightleftharpoons \mathrm{Be}_{\mathrm{III}}\right)$ into account, in which $\mathrm{V}_{\mathrm{III}}$ represents $\mathrm{Ga}$ and In vacancies, to explain the experimental data. Their simulation results suggested that the temperature dependence of group-III self-interstitial and of group-III vacancy effective diffusion coefficients are different. Specifically, for temperatures above $780^{\circ} \mathrm{C}$, group-III interstitial diffusion dominates the group-III vacancy diffusion, while below $780^{\circ} \mathrm{C}$ the situation is reversed. Even though this model dispenses with the local thermodynamical equilibrium assumption and is more realistic than previous models that only considered the kick-out mechanism, it remains a purely phenomenological model: the choice of the mechanism and parameters is only based on fitting.

A number of authors have also proposed different charge states for the diffusion mechanism: specifically, the charge states of $+1,+2$ for Ga and In self-interstitials, $0,-1$ for $\mathrm{Ga}$ and $\mathrm{In}$ vacancies, $0,+1$ for Be interstitial 
and -1 for Be substitutional were proposed ${ }^{4-16}$. These charge states are either inferred from works on GaAs or chosen to fit experiments. Some of these proposed charge states are also counter-intuitive. For example, it is known that $\mathrm{p}$ dopants in group VI semiconductors such as $\mathrm{Mg}$ interstitials in $\mathrm{Si}$ are positively charged, and thus a similar charge state should be expected for $\mathrm{Be}^{17}$. This discrepancy provides the motivation for an independent $a b$ initio determination of the charge state of Be in the InGaAs system, which we will describe in section III C. A realistic model should explain experimental data obtained under different annealing conditions in one consistent way and based on as few assumptions as possible. To the best of our knowledge, such a model is still lacking.

In this work, a comprehensive and physically-based model of diffusion behavior of grown-in Be in InGaAs is presented. To select possible mechanisms for the Be diffusion, we calculated the reaction energies and diffusion barriers of a variety of possible mechanisms using density functional theory. The results suggest that the energies required for the Frank-Turnbull mechanism are much higher than for the kick-out mechanism, and so in the temperature range relevant to experiments, the Frank-Turnbull mechanism can be safely ignored. Furthermore, among the kick-out reactions, the energies required for reactions involving $\mathrm{Ga}$ and In are quite different. Contrary to the previous models, the roles of Ga and In in Be diffusion are different, and these elements ideally should not be lumped together. The influence of As on Be diffusion may not be negligible since the reaction energies for As being kicked out by Be are comparable with Ga/In being kicked out. We then build a diffusion model which based on reaction energies and diffusion barriers calculated from first principles. By implementing this model in the Object Kinetic Monte Carlo simulator (OKMC) $\mathrm{MMonCa},{ }^{18}$ we are able to reproduce experimental data under different annealing temperatures and durations in a consistent way.

\section{METHODOLOGY}

\section{A. Density functional theory}

The calculations were performed using density functional theory ${ }^{19,20}$ with the generalized gradient approximation and the Perdew-Burke-Eznerhof functional (GGA-PBE) $^{21}$ as implemented in Vienna ab initio simulation package (VASP). ${ }^{22-24}$ The projector augmented wave method (PAW) ${ }^{25,26}$ was used to describe the interaction between the atomic cores and electrons. The valence configurations of the atoms were: Arsenic (As) $4 s^{2} 4 p^{3}$, Gallium (Ga) $4 s^{2} 4 p^{1}$, Indium (In) $5 s^{2} 5 p^{1}$ and Beryllium (Be) $2 s^{2} 2 p^{0}$. A $2 \times 2 \times 2$ Monkhorst-Pack ${ }^{27}$ $\mathrm{k}$-point mesh and a cutoff of $400 \mathrm{eV}$ were used for structure optimization. Atomic positions and cell vectors, where applicable, were relaxed using the conjugate gradient (CG) algorithm until all force components were less than $0.01 \mathrm{eV}^{-1}$. The single-point energy calculations on the structures, relaxed using the cutoff of $400 \mathrm{eV}$ and $2 \times 2 \times 2$ k-points, were performed with $6 \times 6 \times 6$ $\mathrm{k}$-points to achieve converged defect formation energies and diffusion barriers. A tetrahedron method with Blöchl ${ }^{25}$ corrections was used for the partial occupancies. The density-of-state (DOS) calculations were performed using $5 \times 5 \times 5 \mathrm{k}$-points meshes. The energy barrier for $\mathrm{Be}, \mathrm{Ga}$ and In-atom diffusion were calculated using the climbing-image nudged elastic band (CI-NEB) method; ${ }^{28,29}$ the force tolerance in the CI-NEB calculations was $0.05 \mathrm{eV}^{-1}$.

To ensure that the simulation cell is of size amenable to the calculations, we used the stoichiometry $\mathrm{In}_{0.5} \mathrm{Ga}_{0.5} \mathrm{As}_{1}$ (abbreviated in the following as InGaAs), as was done in previous works. ${ }^{30,31}$ The simulation cell size of about $12.0 \times 12.0 \times 11.9 \AA$ was used and is sufficient to neglect inter-cell interactions of the defects. The geometries of doped-InGaAs are fully relaxed. The CINEB calculations are performed under fixed cell. The charges on atoms of pure and doped InGaAs crystal are calculated using Bader analysis ${ }^{32}$.

\section{B. Kinetic Monte Carlo simulation}

The Be diffusion process was modeled by object kinetic Monte Carlo using the MMonCa code ${ }^{18}$. In the framework of MMonCa, the diffusion process is composed of succeeding events, either reaction or migration, which occur at different rates. In this study, we consider only kick-out reactions, surface trappings or injections, migrations and transitions between charge states. Once reactants approach each other within the reaction distance, the reaction will occur with the probability

$$
P=\exp \left(-\frac{E_{r e}}{k_{B} T}\right)
$$

when $E_{r e}$ is positive, otherwise the probability is $1 . E_{r e}$ is the reaction barrier, $k_{B}$ is the Boltzmann constant and $T$ is the temperature. In this study, such values are calculated according to the transition state theory. The interface between air and the InGaAs sample is the sink and the source of all self-interstitials: $\mathrm{Ga}_{\mathrm{i}}, \mathrm{In}_{\mathrm{i}}$; the trapping rate and injection rate are also determined by Eq. 1

Self-interstitials and the Be interstitial can migrate in random directions with a fixed migration distance $\lambda$, while substitutional Be atoms are assumed to be immobile. The use of a fixed migration distance is justified by the crystal structure of InGaAs where elementary diffusion steps have all similar lengths. The migration rate is computed as

$$
\nu_{m}=\nu_{m}^{0} \exp \left(-\frac{E_{m}}{k_{B} T}\right)
$$

where $\nu_{m}^{0}$ is the migration attempt frequency; $E_{m}$ is the migration barrier. 
Point defects in semiconductors usually have various electronic states. If we assume that a point defect $\mathrm{X}$ can be in three different charge states for example, singly negative, neutral, singly positive, we will denote this point defect as $X^{j}$, with $j=-, 0,+$. Then the relative concentrations are

$$
\frac{\left[X^{j}\right]}{\left[X^{j+1}\right]}=\frac{g^{j}}{g^{j+1}} \exp \left(\frac{e_{F}-e(j+1, j)}{k_{B} T}\right),
$$

where $g^{j}$ stands for the degeneracy factor, $e_{F}$ is the Fermi level and $e(j+1, j)$ represents the energy level associated to the charge transition between $X^{j}$ and $X^{j+1}$. In the MMonCa framework, a point defect can transform between its different charge states. The transition rate is determined by Eq. 3. In fact, charge state transformation is much faster than the diffusion process, so the relative concentration of charge states is almost in equilibrium everywhere.

So far we have considered only thermal Brownian motion. In our case, doping will introduce an electric field, which will introduce an additional driving force into the diffusion equation. We also need to consider this term. In the MMonCa framework, the ratio between the migration frequency along the electric field and opposite to it for a point defect with charge nq is

$$
\frac{\nu_{m,+}}{\nu_{m,-}}=\exp \left(\frac{n q \varepsilon \lambda}{k_{B} T}\right)
$$

where $n$ is the charge number, $q$ is the elementary charge, $\varepsilon$ is the electric field intensity and $\lambda$ is the migration distance.

Our simulation box has dimensions of $1202 \times 15 \times 15$ $\mathrm{nm}$ with periodic boundary conditions along the $y$ and $z$ directions. The initial Be dopant atoms are all set to the substitutional state and Ga or In interstitials are set at their equilibrium concentrations. The Be concentration profiles are extracted after annealing durations corresponding to those used in the experiments have passed.

\section{RESULTS AND DISCUSSION}

\section{A. Comparison between different diffusion mechanisms: DFT energetics}

The following types of defects and elementary migration steps in Be-doped InGaAs were considered in DFT calculations: (a) migration of an interstitial atom $\left(\mathrm{Be}_{\mathrm{i}}\right.$, $\mathrm{Ga}_{\mathrm{i}}$ and $\mathrm{In}_{\mathrm{i}}$ ) to a neighboring interstitial site, (b) movement of a Be atom from a substitutional site $\left(\mathrm{Be}_{\mathrm{As}}\right.$ : $\mathrm{Be}$ at an $\mathrm{As}$ site, $\mathrm{Be}_{\mathrm{Ga}}$ : $\mathrm{Be}$ at a $\mathrm{Ga}$ site or $\mathrm{Be}_{\mathrm{In}}$ : $\mathrm{Be}$ at an In site) to an interstitial site leaving either $\mathrm{As}$, Ga or In vacancy $\left(\mathrm{V}_{\mathrm{As}}, \mathrm{V}_{\mathrm{Ga}}\right.$ and $\mathrm{V}_{\mathrm{In}}$ )(Frank-Turnbull mechanism) and (c) movement of a $\mathrm{Be}_{\mathrm{i}}$ atom to a substitutional site by displacing $\mathrm{Ga} / \mathrm{In} / \mathrm{As}$ to an interstitial site (kick-out mechanism).
TABLE I: Energy required for Be atom diffusion from a substitutional site $\left(\mathrm{Be}_{\mathrm{As}}, \mathrm{Be}_{\mathrm{Ga}}\right.$ or $\left.\mathrm{Be}_{\mathrm{In}}\right)$ to an interstitial

\begin{tabular}{|c|c|}
\hline Reaction & Reaction energy $(\mathrm{eV})^{1}$ \\
\hline InGaAs $-\mathrm{Be}_{\mathrm{As}} \rightarrow$ InGaAs$-\mathrm{Be}_{\mathrm{i}} \mathrm{V}_{\mathrm{As}}$ & 0.87 \\
\hline $\mathrm{InGaAs}-\mathrm{Be}_{\mathrm{Ga}} \rightarrow \mathrm{InGaAs}-\mathrm{Be}_{\mathrm{i}} \mathrm{V}_{\mathrm{Ga}}$ & 2.50 \\
\hline InGaAs $-\mathrm{Be}_{\text {In }} \rightarrow$ InGaAs $-\mathrm{Be}_{\mathrm{i}} \mathrm{V}_{\text {In }}$ & 2.54 \\
\hline
\end{tabular}
site (leaving $\mathrm{V}_{\mathrm{As}}, \mathrm{V}_{\mathrm{Ga}}$ or $\mathrm{V}_{\mathrm{In}}$ ) calculated by DFT.

TABLE II: Reaction energy required for an interstitial Be atom to migrate to a lattice site of InGaAs by kicking out a Ga, In or As atom to an interstitial site

\begin{tabular}{|c|c|}
\hline Reaction & $\overline{\text { Reaction energy }(\mathrm{eV})}$ \\
\hline $\mathrm{InGaAs}+\mathrm{Be}_{\mathrm{i}} \rightarrow \operatorname{InGaAs}-\mathrm{Be}_{\mathrm{Ga}}+\mathrm{Ga}_{\mathrm{i}}\left(\right.$ site $\left._{1}\right)$ & -0.63 \\
\hline $\mathrm{InGaAs}+\mathrm{Be}_{\mathrm{i}} \rightarrow \operatorname{InGaAs}-\mathrm{Be}_{\mathrm{Ga}}+\mathrm{Ga}_{\mathrm{i}}\left(\operatorname{site}_{2}\right)$ & -0.72 \\
\hline $\mathrm{InGaAs}+\mathrm{Be}_{\mathrm{i}} \rightarrow \operatorname{InGaAs}-\mathrm{Be}_{\mathrm{In}}+\operatorname{In}_{\mathrm{i}}\left(\right.$ site $\left._{1}\right)$ & 0.31 \\
\hline $\operatorname{InGaAs}+\mathrm{Be}_{\mathrm{i}} \rightarrow \operatorname{InGaAs}-\mathrm{Be}_{\mathrm{In}}+\operatorname{In}_{\mathrm{i}}\left(\operatorname{site}_{2}\right)$ & 0.11 \\
\hline $\mathrm{InGaAs}+\mathrm{Be}_{\mathrm{i}} \rightarrow \operatorname{InGaAs}-\mathrm{Be}_{\mathrm{As}}+\mathrm{As}_{\mathrm{i}}\left(\right.$ site $\left._{1}\right)$ & 0.37 \\
\hline $\mathrm{InGaAs}+\mathrm{Be}_{\mathrm{i}} \rightarrow \operatorname{InGaAs}-\mathrm{Be}_{\mathrm{As}}+\mathrm{As}_{\mathrm{i}}\left(\mathrm{site}_{2}\right)$ & 0.17 \\
\hline
\end{tabular}
calculated by DFT.

In Table I, we list the reaction energies for a Be atom diffusion from a $\mathrm{Be}_{\mathrm{As}}, \mathrm{Be}_{\mathrm{Ga}}$ or $\mathrm{Be}_{\mathrm{In}}$ substitutional site to an interstitial site leaving either $\mathrm{V}_{\mathrm{As}}, \mathrm{V}_{\mathrm{Ga}}$ or $\mathrm{V}_{\mathrm{In}}$, respectively (see Fig. 1). The positive reaction energies mean that the diffusion of a Be-atom from a substitutional site to an interstitial site is an endothermic reaction. Table II lists reaction energies for various kick-out reactions, and corresponding configurations are shown in Fig. 2. In these reactions, the lattice $\mathrm{Ga}$, In, and As atoms can be displaced by a Be atom into two kinds of interstitial sites (site1 and site 2), as shown in Fig. 2. Comparing with reaction energies needed for the kick-out mechanism (Table II), Frank-Turnbull mechanism requires much higher energies. We can therefore conclude that the kick-out mechanism is the dominant mechanism, at least in the annealing temperature range $\left(700^{\circ} \mathrm{C}-900^{\circ} \mathrm{C}\right)$ in experiments.

We also note from Table II that In and Ga appear to have different roles. For simplification, previous models all treated In and Ga as the same idealized group-III element. However, as can be seen from Table II, their properties are quite different: the kick-out of Ga by a Be interstitial is exothermic while the kick-out of In by a Be interstitial is endothermic. It is worth noting that the reaction energies of kick-out of As by a Be interstitial is comparable with kick-out of the III-group elements, which is ignored by previous models. However the role of As is complicated by the swap reaction (see Fig. 3e), so that full picture on how As influences Be diffusion is difficult to work out. 

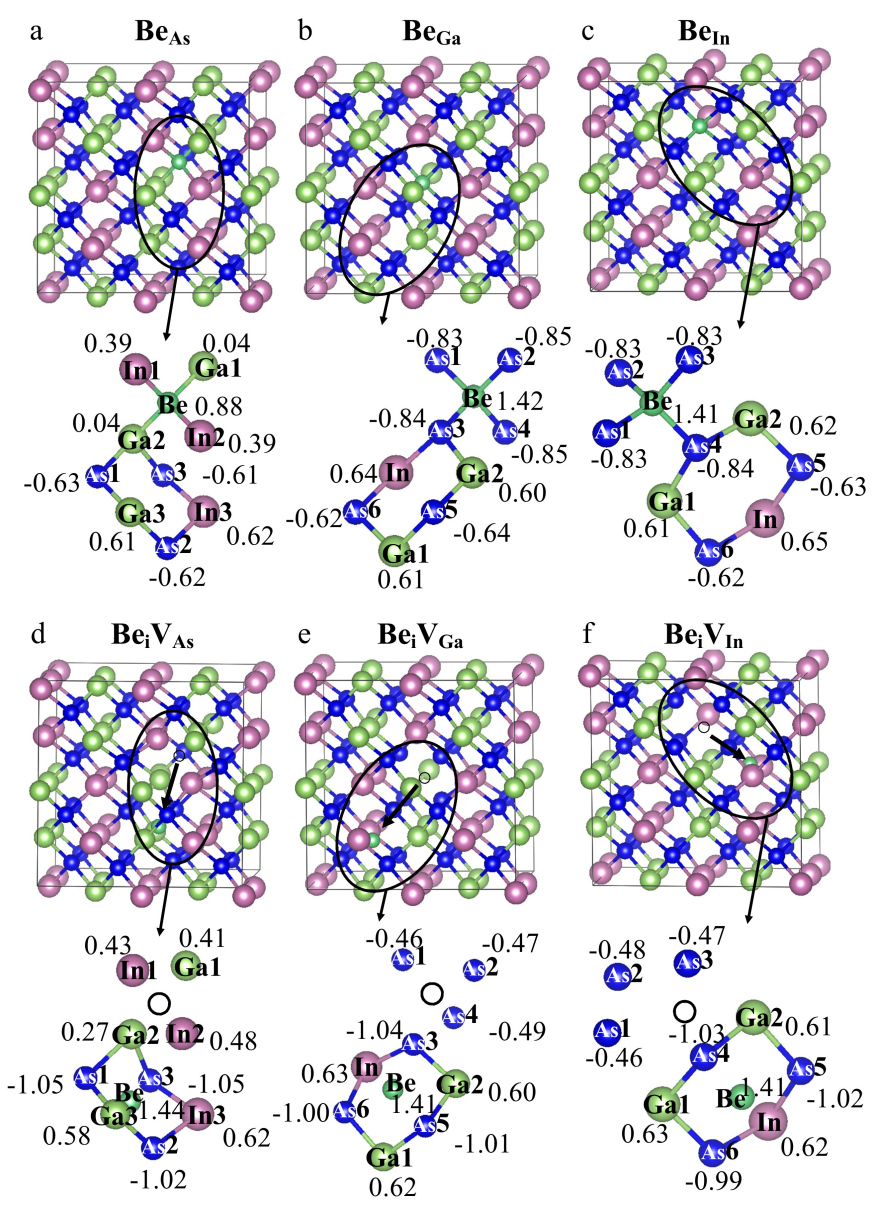

Fig. 1: Structures of (a) $\mathrm{Be}_{\mathrm{As}}$, (b) $\mathrm{Be}_{\mathrm{Ga}}$ and (c) $\mathrm{Be}_{\mathrm{In}}$ doped InGaAs. (d), (e) and (f) are the structures of doped InGaAs after the $\mathrm{Be}_{\mathrm{As}}, \mathrm{Be}_{\mathrm{Ga}}$ and $\mathrm{Be}_{\mathrm{In}}$ atoms moved to an interstitial site leaving $\mathrm{V}_{\mathrm{As}}, \mathrm{V}_{\mathrm{Ga}}$ and $\mathrm{V}_{\mathrm{In}}$, respectively. Insets show the Be atom and its neighbouring atoms with Bader charges. Violet spheres - In, large green spheres - Ga, blue spheres - As and small green spheres Be atom. Visualization here and elsewhere is by VESTA ${ }^{33}$.

\section{B. Diffusion paths and barriers: DFT kinetics}

The higher reaction energies calculated in section III A suggest that the Frank-Turnbull mechanism is not important. The calculated diffusion barriers for the $\mathrm{Be}_{\mathrm{Ga}}$ and $\mathrm{Be}_{\text {In }}$ diffusions to an interstitial site are 3.37 and $3.40 \mathrm{eV}$ (see Fig. 3a, b, c and d, respectively). The high reaction energies and barriers allow us to discard both reactions. For the diffusion of the $\mathrm{Be}_{\mathrm{As}}$ to an interstitial site leaving $\mathrm{V}_{\mathrm{As}}$, the path is more complex: the $\mathrm{Be}_{\mathrm{As}}$ swaps the position with a neighbouring Ga-atom (see Fig. 3e, f) instead of migrating to an interstitial site. The reaction energy for this swapping reaction is $-0.68 \mathrm{eV}$ and the barrier is $1.11 \mathrm{eV}$. This result suggests that $\mathrm{Be}_{\mathrm{As}}$ is an unstable configuration for the Be dopant. Therefore there will only be transient occupation of As sites by Be at high
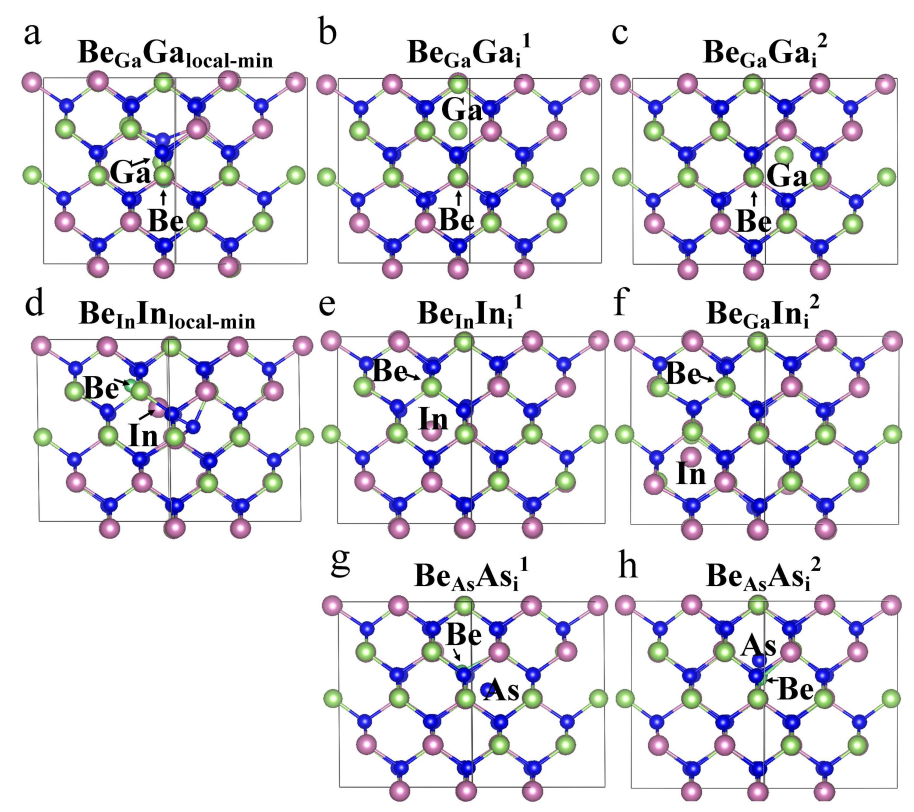

Fig. 2: Structure of InGaAs with (a,b,c) a substitutional Be atom and $\mathrm{a}$ Ga atom at an interstitial site (local minimum, site1 and site2, respectively) and (d,e,f) a substitutional Be atom and an In atom at an interstitial site (local minimum, site1 and site2, respectively) and $(\mathrm{g}, \mathrm{h})$ a substitutional Be atom and an As atom at an interstitial site (site1 and site2, respectively)

temperatures.

We have also calculated the diffusion paths and barriers for the kick-out mechanism. Both the kick-out of Ga and the kick-out of In can be divided into two steps separated by a local minimum, as shown in Fig. 4 . In the case of Ga being kicked out by the Be interstitial, barriers for steps 1 and 2 are 0.42 and $0.55 \mathrm{eV}$ respectively; whereas for the In being kicked out, barriers are 0.70 and $0.43 \mathrm{eV}$ respectively. For the kick-out of a Ga atom, the energy barrier for step 1 is lower than for the kick-out of In. The local energy minima suggest the existence of quasi-stable Be-Ga and Be-In split-interstitial structures or dumbbell configurations. In contrast, we did not observe any local energy minimum for the As atom being kicked out (see Fig. 5), and the diffusion barrier is $0.58 \mathrm{eV}$.

It is important to note that although both In and Ga being kicked out can be divided into two steps, the physics are different: for the kick out of $\mathrm{Ga}$, the intermediate state has a higher energy than the final state and therefore will not influence the reaction outcome; for the kick out of In, the intermediate state is more like a sink because its energy is lower than that of both the initial state and the final state. This emphasizes the importance of treating In and Ga separately.

The calculated diffusion barriers for the migration of $\mathrm{Be}_{\mathrm{i}}, \mathrm{Ga}_{\mathrm{i}}$ and $\mathrm{In}_{\mathrm{i}}$ from an interstitial site to a neighboring interstitial site in InGaAs are 0.63, 0.93 and $0.98 \mathrm{eV}$, respectively (see Fig. 6). We note that the diffusion paths 


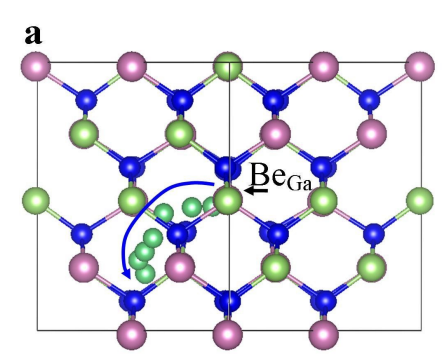

b
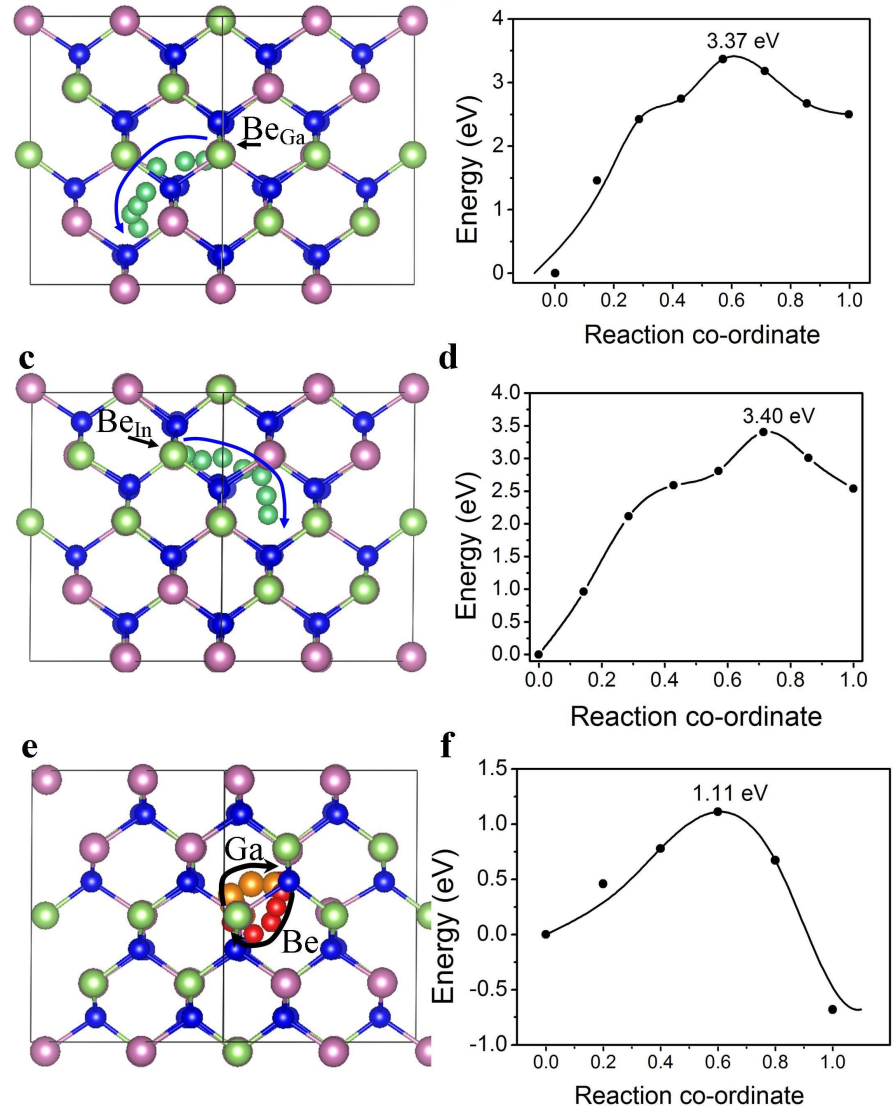

Fig. 3: Diffusion path of (a) $\mathrm{Be}_{\mathrm{Ga}}$ to $\mathrm{Be}_{\mathrm{i}}$ leaving $\mathrm{V}_{\mathrm{Ga}}$ vacancy (b) $B e_{\text {In }}$ to $\mathrm{Be}_{\mathrm{i}}$ leaving $\mathrm{V}_{\text {In }}$ vacancy and (c) $\mathrm{Be}_{\mathrm{As}}$ to $\mathrm{Be}_{\mathrm{Ga}}$ and $\mathrm{Ga}_{\mathrm{Ga}}$ to $\mathrm{Ga}_{\mathrm{As}}$ in InGaAs. (b), (d) and (f) are the calculated potential energy curve along the diffusion path for diffusion in (a), (c) and (e), respectively. Be at $\mathrm{Ga}$, In and $\mathrm{As}$ site at equilibrium corresponds to 0.0 on the abscissa. Be after diffusion at equilibrium corresponds to 1.0. The solid line in panels (b, d and f) is given to guide the eyes.

of $\mathrm{Ga}_{\mathrm{i}}$ and $\mathrm{In}_{\mathrm{i}}$ are straight and symmetric whereas the diffusion path of $\mathrm{Be}_{\mathrm{i}}$ is curved and asymmetric.

\section{Charge state analysis: DFT}

The average Bader charges on $\mathrm{As}, \mathrm{Ga}$ and In atom in pure InGaAs crystal are $-0.64 e,+0.61 e$ and $+0.67 e$, respectively as shown in Fig. 7c. There is one symmetryunique interstitial binding site in the InGaAs crystal for Be atom insertion $\left(\mathrm{Be}_{\mathrm{i}}\right)$ as shown in Fig. $7 \mathrm{~b}, \mathrm{~d}$. The Be atom is on top of the plane formed by three nearest As atoms and equidistant from each As atoms. The calculated Bader charge on $\mathrm{Be}_{\mathrm{i}}$ atom is +1.35 e (see Table III and Fig. $7 \mathrm{~d}$ ) in the equilibrium position but becomes $1.15 e$ in the transition state for diffusion. By examining the Bader charges of the neighboring As, Ga, and In a

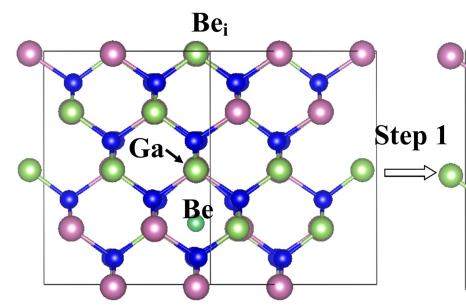

$\mathrm{b}$
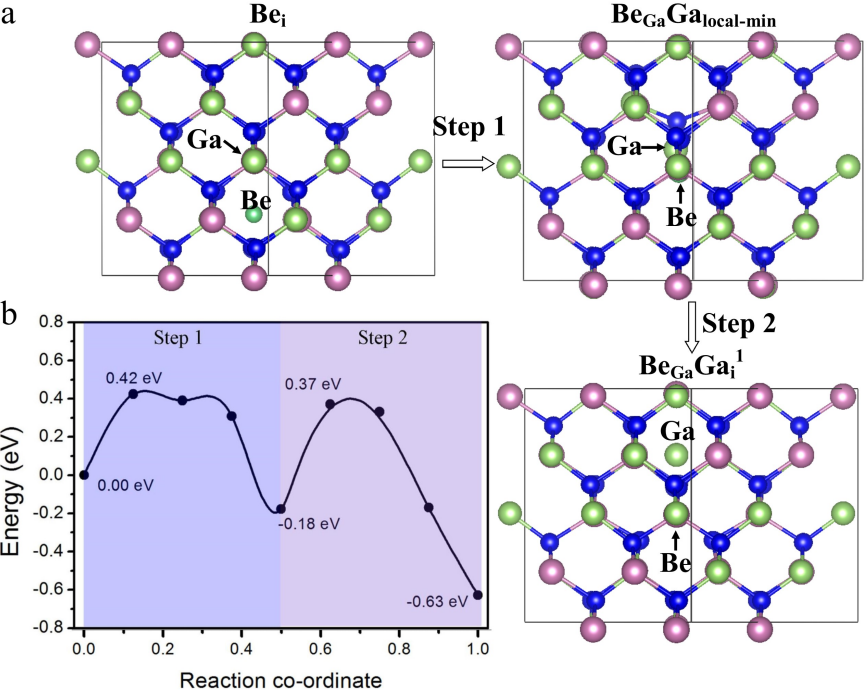

c
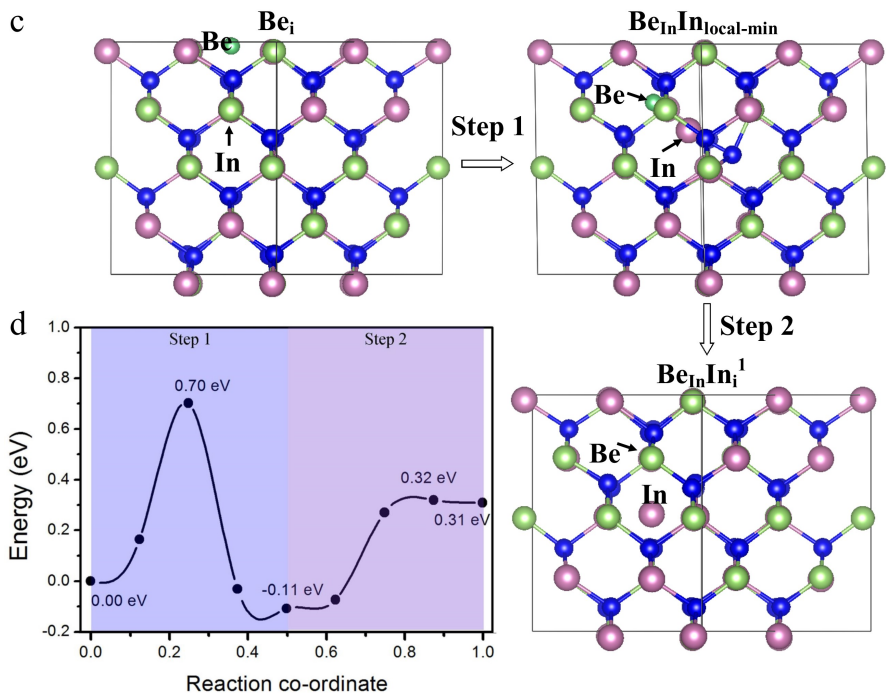

Fig. 4: Steps of $\mathrm{Be}_{\mathrm{i}}$ atom diffusion to (a) $\mathrm{Be}_{\mathrm{Ga}}$ by kicking out the Ga atom and (c) $\mathrm{Be}_{\mathrm{In}}$ by kicking out the In atom to an interstitial site. The calculated potential energy curve along the diffusion path of the $\mathrm{Be}_{\mathrm{i}}$ to (b) $\mathrm{Be}_{\mathrm{Ga}}$ by kicking out the Ga atom to an interstitial site and (d) $\mathrm{Be}_{\mathrm{In}}$ by kicking out the In atom to an interstitial site. Be atom at an interstitial site $\left(\mathrm{Be}_{\mathrm{i}}\right)$ corresponds to 0.0 and $\mathrm{Be}$ at either $\mathrm{Ga}$ or In site $\left(\mathrm{Be}_{\mathrm{Ga}}\right.$ or $\left.\mathrm{Ge}_{\mathrm{In}}\right)$ with either Ga or In atom moved to an interstitial site corresponds to 1.0 on the abscissa. The solid line in panels ( $\mathrm{b}$ and $\mathrm{d}$ ) is given to guide the eyes.

atoms, we find that the inserted $\mathrm{Be}_{\mathrm{i}}$ atom donates electron density to its neighbors. The Bader charges on the As atoms (see Fig. 7c, d) significantly changes upon Be insertion, going from $-0.64 e$ to $-1.01 e$ suggesting that the dominant electron donation is from the Be atom to neighbouring As atoms. There are two symmetry-unique interstitial binding sites in InGaAs for $\mathrm{Ga}\left(\mathrm{Ga}_{\mathrm{i}}\right)$ and In $\left(\mathrm{In}_{\mathrm{i}}\right)$ atom insertion. The total energy difference between the two $\mathrm{Ga}_{\mathrm{i}}$ sites is $0.01 \mathrm{eV}$ whereas for $\mathrm{In}_{\mathrm{i}}$ total energy 


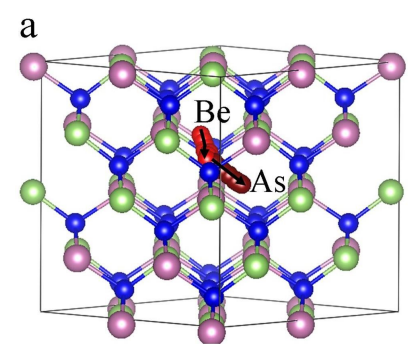

b

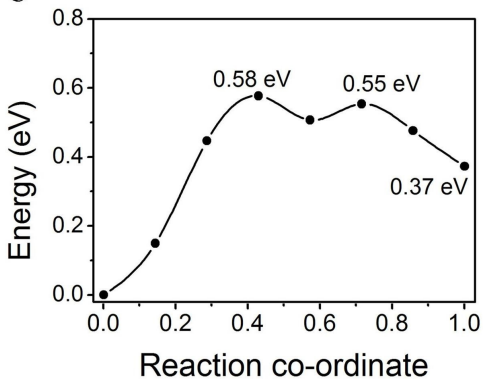

Fig. 5: (a) Diffusion path for As kick-out by $\mathrm{Be}_{\mathrm{i}}$ in InGaAs and (b) the calculated potential energy curve along the diffusion path. The Be atom at an interstitial site corresponds to 0.0; and Be atom at As site and As atom at an interstitial site after the kick out corresponds to 1.0 on the abscissa. The solid line is given to guide the eyes.

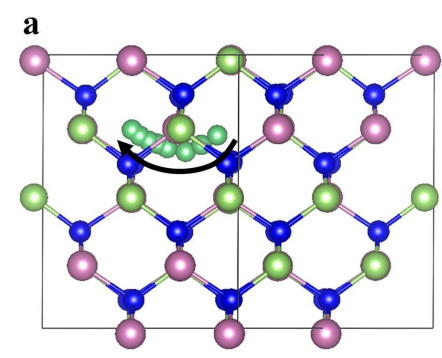

b

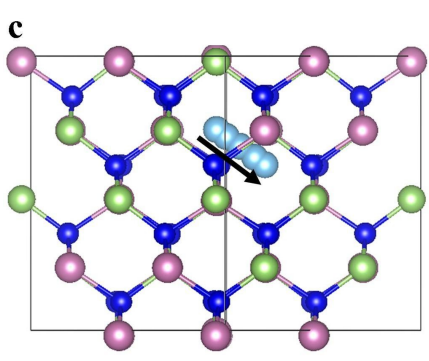

d
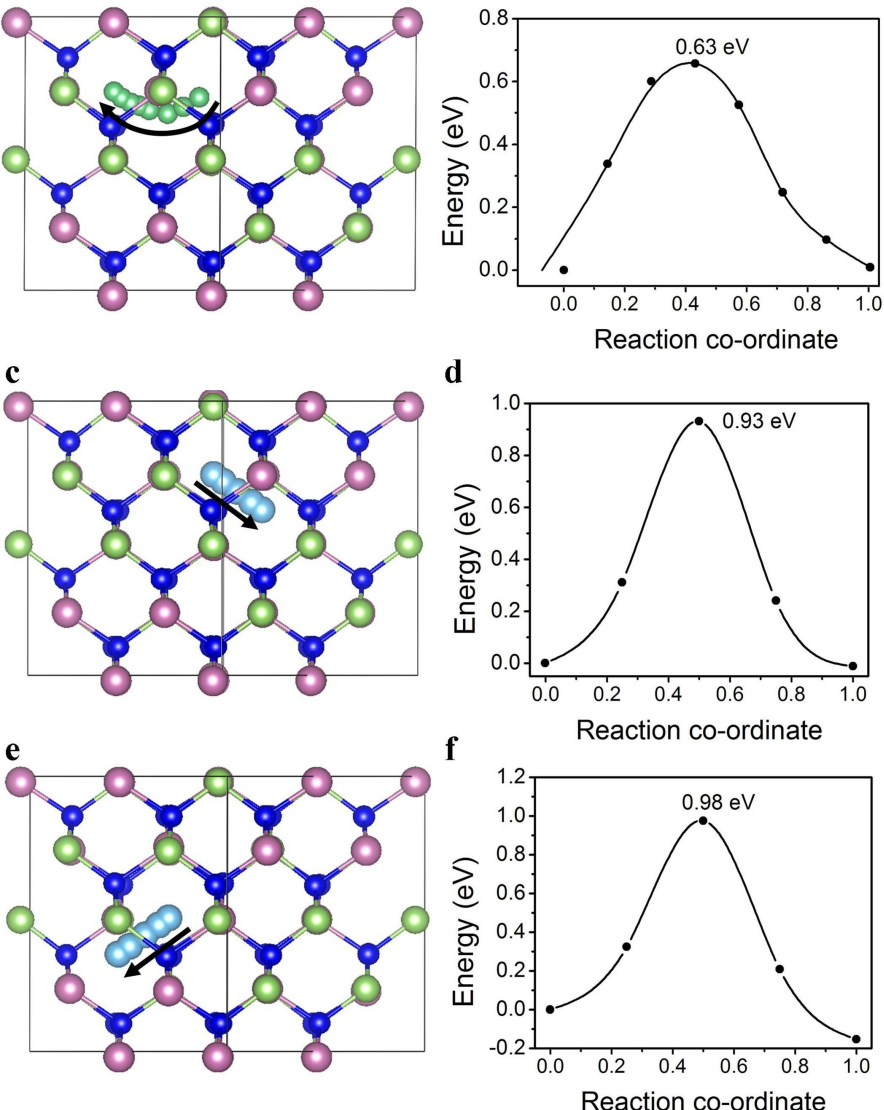

Fig. 6: (a) Diffusion path of (a) $\mathrm{Be}_{\mathrm{i}}$, (c) $\mathrm{Ga}_{\mathrm{i}}$ and (e) $\mathrm{In}_{\mathrm{i}}$ in InGaAs. (b), (d) and (f) are the calculated potential energy curves along the diffusion path in (a), (c) and (e), respectively. Be, Ga and In at an interstitial site at equilibrium corresponds to 0.0. Be, Ga and In at another interstitial site after diffusion at equilibrium corresponds to 1.0 on the abscissa. The solid line in panels (b, $d$ and $f$ ) is given to guide the eyes.
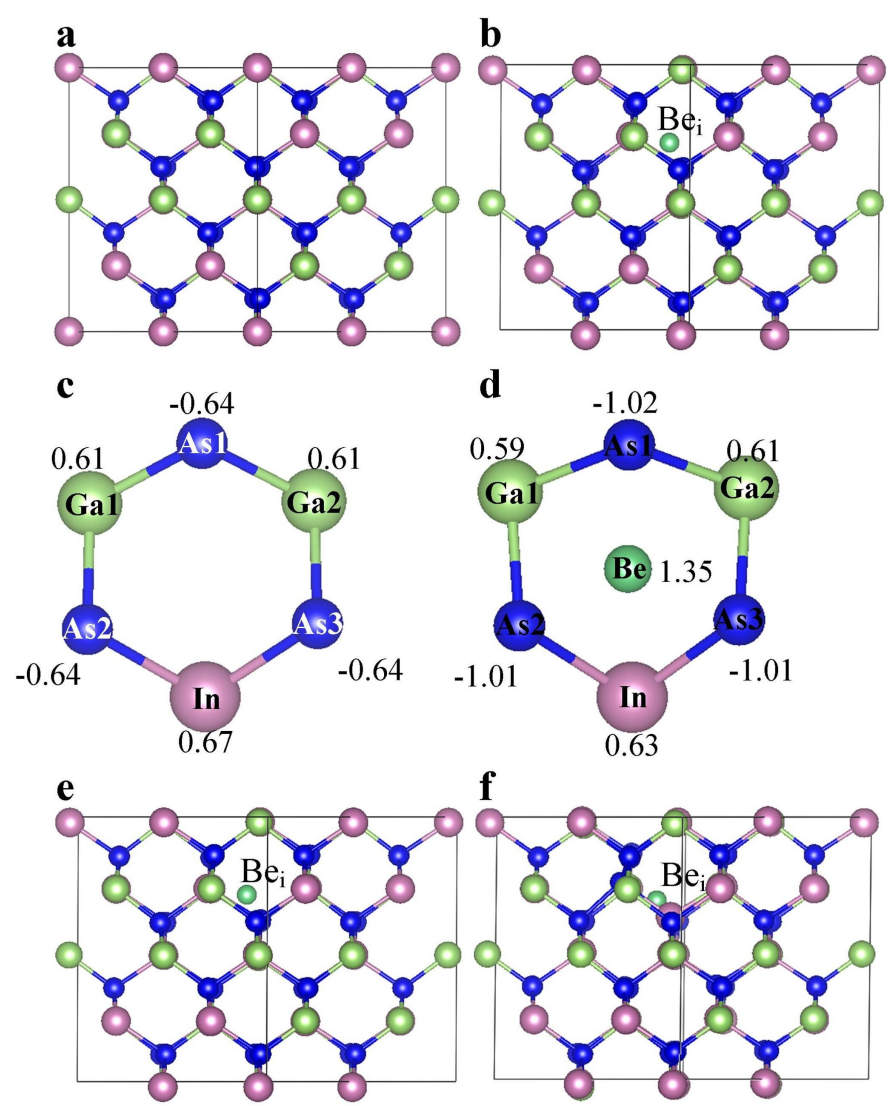

g
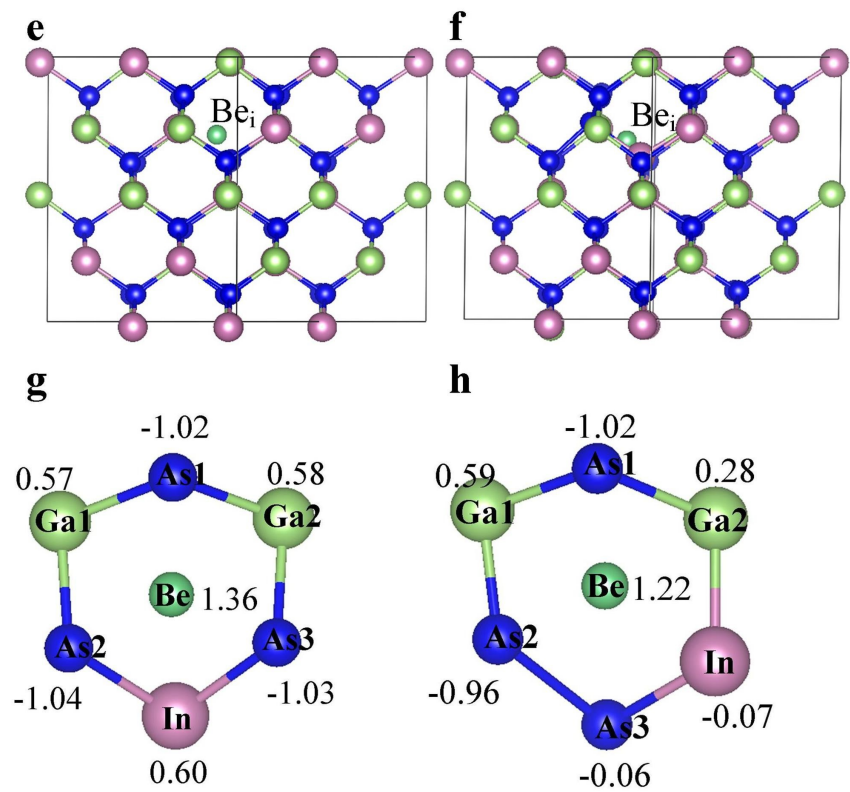

h

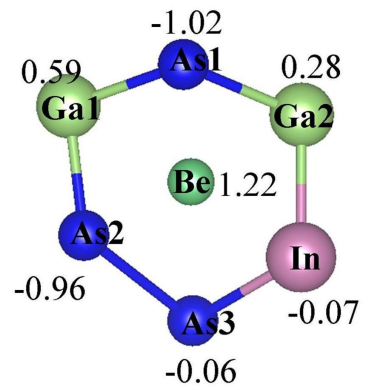

Fig. 7: (a) Pure and (b) $\mathrm{Be}_{\mathrm{i}}$ doped InGaAs

$\left(\mathrm{Be}_{\mathrm{i}}-\mathrm{InGaAs}\right)$. (e) $\mathrm{Be}_{\mathrm{i}}-\mathrm{InGaAs}$ with charged supercell $(-1 \mathrm{e})$ and (f) $\mathrm{Be}_{\mathrm{i}}$-InGaAs with flipping the position of As with In atom near $\mathrm{Be}_{\mathrm{i}}$. Atoms domain with Bader charges in (c) pure, (d) $\mathrm{Be}_{\mathrm{i}}-\mathrm{InGaAs}$, (g) $\mathrm{Be}_{\mathrm{i}}-\mathrm{InGaAs}$ with charged supercell and $(\mathrm{h}) \mathrm{Be}_{\mathrm{i}}-\mathrm{InGaA}$ with flipping the position of In with As atom near $\mathrm{Be}_{\mathrm{i}}$.

difference is $0.15 \mathrm{eV}$. The calculated Bader charges on $\mathrm{Ga}_{\mathrm{i}}$ atoms in the two sites are $0.36 e$ and 0.28 e respectively in the equilibrium position and $0.31 e$ in the transition state for diffusion. Similarly, charges on $\mathrm{In}_{\mathrm{i}}$ in the two sites are $0.43 e$ and $0.37 e$, respectively, in the equilibrium position and $0.40 e$ in the transition state for diffusion.

The calculated Bader charges on the $\mathrm{Be}_{\mathrm{As}}, \mathrm{Be}_{\mathrm{Ga}}$ and $\mathrm{Be}_{\text {In }}$ are $+0.88 e,+1.42 e$ and $+1.41 e$, respectively. The 
TABLE III: Calculated Bader charges on Be, Ga and In atoms in doped InGaAs.

\begin{tabular}{cc}
\hline Atom type & Bader charge $[e]$ \\
\hline $\mathrm{Be}_{\mathrm{i}}$ & 1.35 \\
$\mathrm{Ga}_{\mathrm{i}}$ & $0.36(0.28)$ \\
$\mathrm{In}_{\mathrm{i}}$ & $0.43(0.37)$ \\
$\mathrm{Be}_{\mathrm{As}}$ & 0.88 \\
$\mathrm{Be}_{\mathrm{Ga}}$ & 1.42 \\
$\mathrm{Be}_{\mathrm{In}}$ & 1.41 \\
$\mathrm{Be}_{\mathrm{i}} \mathrm{V}_{\mathrm{As}}$ & 1.44 \\
$\mathrm{Be}_{\mathrm{i}} \mathrm{V}_{\mathrm{Ga}}$ & 1.41 \\
$\mathrm{Be}_{\mathrm{i}} \mathrm{V}_{\mathrm{In}}$ & 1.41 \\
$\mathrm{Be}_{\mathrm{Ga}} \mathrm{Ga}_{\mathrm{i}}^{1}$ & 1.42 \\
$\mathrm{Be}_{\mathrm{Ga}} \mathrm{Ga}_{\mathrm{i}}^{2}$ & 1.40 \\
$\mathrm{Be}_{\mathrm{In}} \mathrm{In}_{\mathrm{i}}^{1}$ & 1.38 \\
$\mathrm{Be}_{\mathrm{In}} \mathrm{In}_{\mathrm{i}}^{2}$ & 1.41 \\
\hline
\end{tabular}

$\mathrm{Be}_{\mathrm{As}}$ atom donates electrons to $\mathrm{Ga}$ and In whereas $\mathrm{Be}_{\mathrm{Ga}}$ and $\mathrm{Be}_{\mathrm{In}}$ donate electron to the As atoms (see Fig. 1). Calculated Bader charges on the Be atom for $\mathrm{Be}_{\mathrm{i}} \mathrm{V}_{\mathrm{As}}$, $\mathrm{Be}_{\mathrm{i}} \mathrm{V}_{\mathrm{Ga}}$ and $\mathrm{Be}_{\mathrm{i}} \mathrm{V}_{\text {In }}$ configurations are $+1.44 e,+1.41 e$ and +1.41 e, respectively (see Table III and Fig. 1). The total charges on the atoms bonded to the Be atom in $\mathrm{Be}_{\mathrm{As}}, \mathrm{Be}_{\mathrm{Ga}}$ and $\mathrm{Be}_{\mathrm{In}}$ doped InGaAs are $+0.86 e,-3.37 e$ and $-3.33 e$ respectively, which change to $+1.59 e,-2.46 e$ and $-2.46 e$, respectively, when the Be atom diffuses to the interstitial site.

To investigate the kick-out mechanism, we have replaced either an Ga or an In atom with a Be atom from the interstitial site and placed that $\mathrm{Ga}$ or In atom in interstitial sites; $\mathrm{Be}_{\mathrm{Ga}} \mathrm{Ga}_{\mathrm{i}}^{1}, \mathrm{Be}_{\mathrm{Ga}} \mathrm{Ga}_{\mathrm{i}}^{2}, \mathrm{Be}_{\mathrm{Ga}} \mathrm{In}_{\mathrm{i}}^{1}, \mathrm{Be}_{\mathrm{Ga}} \mathrm{In}_{\mathrm{i}}^{2}$ (see Fig. 2). The calculated Bader charges on the $\mathrm{Be}$ in configuration $\mathrm{Be}_{\mathrm{Ga}} \mathrm{Ga}_{\mathrm{i}}^{1}, \mathrm{Be}_{\mathrm{Ga}} \mathrm{Ga}_{\mathrm{i}}^{2}, \mathrm{Be}_{\mathrm{In}} \mathrm{In}_{\mathrm{i}}^{1}$ and $\mathrm{Be}_{\mathrm{In}} \mathrm{In}_{\mathrm{i}}^{2}$ are $+1.42 e,+1.40 e,+1.38 e$ and $+1.41 e$, respectively (see Table III).

Like any charge definition, the Bader charge analysis used here is but one definition of atomic charges; it is done based on the topology of the electron density and naturally results in non-integer charge states. These charge states can, however, be assigned to integer charge states and compared to those used in the KMC model by considering the valence shell build-up of the dopant. For $\mathrm{Be}_{\mathrm{i}}$, the Bader charge state of about 1.4e therefore corresponds to the donation of both its $2 s$ electrons to the host structure (specifically, to the conduction band, as shown in Fig. 9 , i.e. $\mathrm{Be}_{\mathrm{i}}$ is a n-type dopant). This is corroborated by the fact that the zero spin state is preserved upon Be insertion (i.e. both valence electrons of $\mathrm{Be}$ are in the conduction band). $\mathrm{Be}_{\mathrm{i}}$ can therefore be assigned a $0-\mathrm{K}$ state of +2 . Likewise, the $\mathrm{Ga}_{\mathrm{i}}$ Bader charge of about $+0.3 e$ suggests that the Ga atom does not lose any of its valence electrons and the charge of $0.3 e$ is due to charge redistribution following bond formation with the host atoms. We can assign to $\mathrm{Ga}_{\mathrm{i}}$ a $0-\mathrm{K}$ state of 0 . Similarly we assign a charge state of 0 to $\mathrm{In}_{\mathrm{i}}$.

Be atoms substituting a $\mathrm{Ga}$ or an In atom $\left(\mathrm{Be}_{\mathrm{Ga}}\right.$ and $\left.\mathrm{Be}_{\mathrm{In}}\right)$ also loose both their valence electrons, except in a

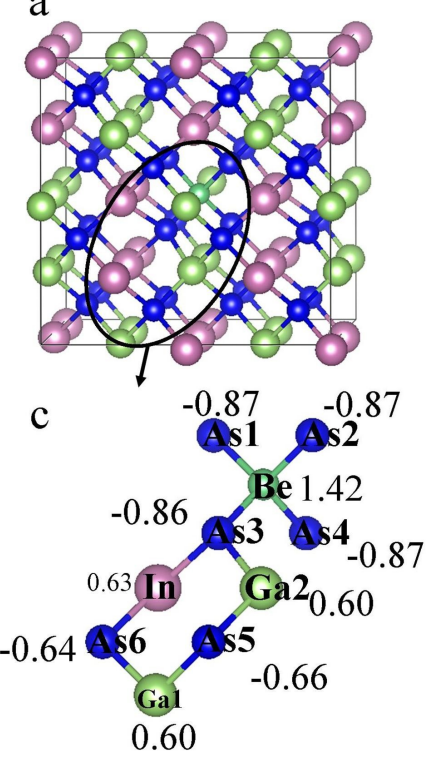

b

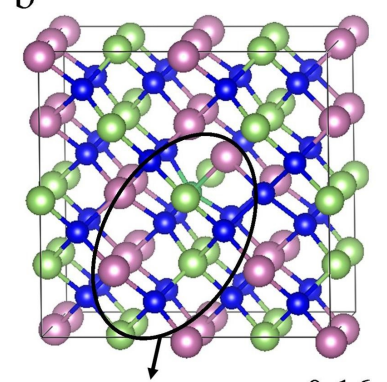

d

As1 In2

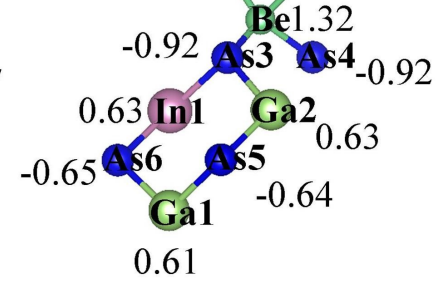

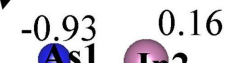

Fig. 8: (a) $\mathrm{Be}_{\mathrm{Ga}}-\mathrm{InGaAs}$ with charged supercell $(-1 e)$ and (b) $\mathrm{Be}_{\mathrm{Ga}}$-InGaAs with flipping the position of As with In atom near $\mathrm{Be}_{\mathrm{Ga}}$. Atoms surrounding the $\mathrm{Be}$ dopant with Bader charges in (c) $\mathrm{Be}_{\mathrm{Ga}}-\mathrm{InGaAs}$ with charged supercell (-1e) and (d) $\mathrm{Be}_{\mathrm{Ga}}-\mathrm{InGaAs}$ with flipping the position of In with As atom near $\mathrm{Be}_{\mathrm{Ga}}$.

this case the electrons occupy states in the valence band, as shown in Fig. 11. This means that substitutional Be atoms play the role of $\mathrm{p}$ dopants but remain positively charged (about $+1.4 e$ ) in the host structure similarly to $\mathrm{Mg}$ doping of $\mathrm{Si}$ or $\mathrm{Ge}^{34,35}$. We have confirmed that the positive charge does not noticeably change even when imposing a negative charge on the supercell (see Fig. 7). We therefore assign $0-\mathrm{K}$ states of +2 to both $\mathrm{Be}_{\mathrm{Ga}}$ and $\mathrm{Be}_{\text {In }}$.

The above charge calculation is done for an ideal ordered crystal, however the real InGaAs is a random alloy. To test the effects due to local disorder and nonstoichiometry, we exchanged the positions of As and In atoms in the vicinity of the $\mathrm{Be}_{\mathrm{i}}$ atom. Fig. $7 \mathrm{~g}$, h shows the resulting structures and atomic charges. This antisite defect does not significantly change the charge state of Be; however, it changes the charge states of the involved As and In atoms to near-neutral. The total energy cost of such an exchange is $1.43 \mathrm{eV}$, so the effect of these configurations can be neglected.

Also, when we exchange the In atom with an As atom near the $\mathrm{Be}_{\mathrm{Ga}}$ site, there are significant differences of charges on the exchanged In and As atoms (see Fig. 8c), but no significant change in the charge state of $\mathrm{Be}_{\mathrm{Ga}}$. Similar to $\mathrm{Be}_{\mathrm{i}}-\mathrm{InGaAs}$, the flipped In and As atoms can have a zero charge state in $\mathrm{Be}_{\mathrm{Ga}}-\mathrm{InGaAs}$. However, the energy required to exchange the In atom with an As atom is $2.06 \mathrm{eV}$ and therefore the presence of such antisite defects can be neglected. The calculations show that 

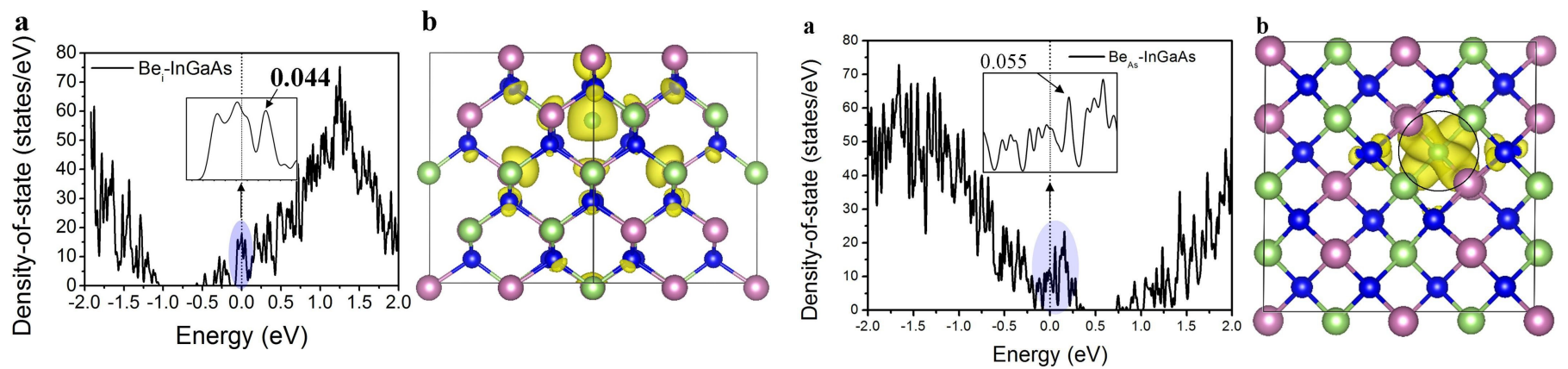

Fig. 9: (a) Total DOS plot of $\mathrm{Be}_{\mathrm{i}}$-doped InGaAs. The Fermi level is set to zero (dotted line). The inset is the zoomed up DOS plot where the energy axis (x-axis) ranges from -0.1 to $0.1 \mathrm{eV}$. (b) The partial (band decomposed) charge density plot of band at $0.044 \mathrm{eV}$ above the Fermi level.

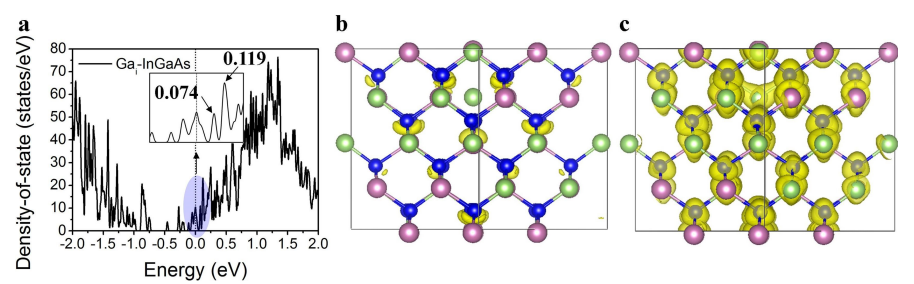

Fig. 10: (a) Total DOS plot of $\mathrm{Ga}_{\mathrm{i}}$-doped InGaAs. The Fermi level is set to zero (dotted line). The inset is the zoomed up DOS plot where the energy axis (x-axis) ranges from -0.2 to $0.2 \mathrm{eV}$. (b) and (c) The partial (band decomposed) charge density of band at 0.074 and $0.119 \mathrm{eV}$ above the Fermi level.

non-stoichiometric effects will surely influence the charge distribution, however, due to their high defect formation energy, they are not expected to have a significant effect on the model.

We emphasize that the above charge state assignment is valid at zero temperature (for which the $a b$ initio calculations are done). At finite temperature, available empty states just above the Fermi level $\left(E_{f}\right)$ will be occupied. If these states are localized on the dopant, this will generate other dopant charge states. We therefore analyse possible charge states relevant for the KMC simulations below. For $\mathrm{Be}_{\mathrm{i}}$, there is a Be-centered state $0.044 \mathrm{eV}$ above $E_{f}$, as shown in Fig. 9. According to the Boltzmann distribution, the partial occupancy of this state is $18 \%$ at $300 \mathrm{~K}$. This suggests that at $300 \mathrm{~K} 18 \%$ of $\mathrm{Be}_{\mathrm{i}}$ atoms will be in state +1 rather than +2 . At temperatures relevant to experiments as well as KMC simulations, i.e. $1100 \mathrm{~K}$, $63 \%$ of $\mathrm{Be}_{\mathrm{i}}$ will be in state +1 . Similarly, we identified a state at $0.119 \mathrm{eV}$ above $E_{f}$ for $\mathrm{Ga}_{\mathrm{i}}$. This state is not strictly Ga-centred but has a significant localization on the $\mathrm{Ga}_{\mathrm{i}}$, as shown in Fig. 10. The occupation probability of this state (and therefore the fraction $\mathrm{Ga}_{\mathrm{i}}^{-}$is $1 \%$ ) at $300 \mathrm{~K}$ and $28 \%$ at $1100 \mathrm{~K}$. For $\mathrm{Be}_{\mathrm{Ga}}$ and $\mathrm{Be}_{\mathrm{In}}$, we did not find low-lying Be-centred states likely to be occupied at finite temperatures.
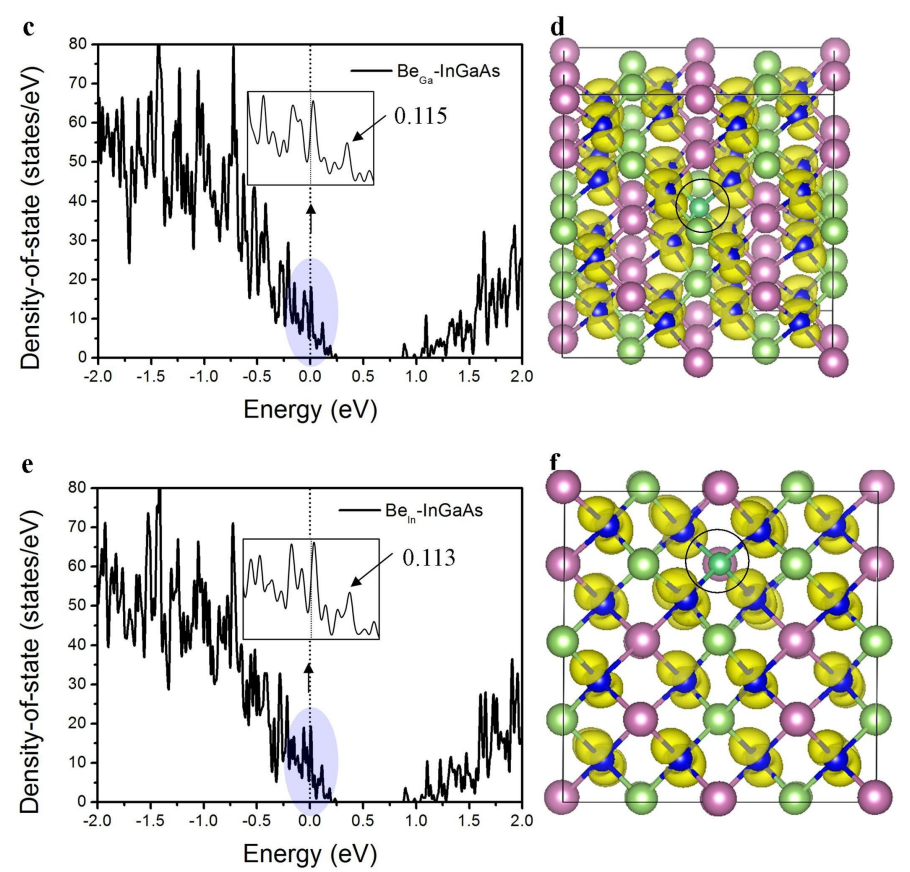

Fig. 11: Total DOS plot of (a) $\mathrm{Be}_{\mathrm{As}}$, (c) $\mathrm{Be}_{\mathrm{Ga}}$ and (e) $\mathrm{Be}_{\text {In }}$ doped InGaAs. The Fermi level is set to zero (dotted line). The insets are the zoomed up DOS plots where the energy axis (x-axis) ranges from -0.2 to 0.2 $\mathrm{eV}$. The partial (band decomposed) charge density plot of bands at 0.055, 0.115 and $0.113 \mathrm{eV}$ above the Fermi level in (b) $\mathrm{Be}_{\mathrm{As}}$, (d) $\mathrm{Be}_{\mathrm{Ga}}$ and (f) $\mathrm{Be}_{\mathrm{In}}$ doped InGaAs, respectively.

\section{Continuum calculations}

Continuum calculations of diffusion in solids are widely used due to their conceptual simplicity and numerical flexibility. However, the complexity grows rapidly with the number of reactions involved. This is the case for Be diffusion in InGaAs where multiple elementary steps contribute to Be diffusion as we have elucidated in the preceding sections. Nevertheless, with a few additional assumptions, continuum calculations can still provide valuable insights as an exploratory tool, as we will demonstrate as follows.

The continuum treatment is based on Fick's law of diffusion. Once the mechanism is assumed, one can write 
down the corresponding differential equations and solve them numerically. The equations parametrically depend on diffusivities and reaction energies, which one can fit to match experimental data. Previous simulations have shown that the simulated Be concentration profile is not very sensitive to the effective diffusivity, but is instead very sensitive to the charge state assumed in the diffusion mechanism. The charge state can therefore be reliably determined from the fit. ${ }^{5}$

Our goal is to explain many experiments using a single, consistent set of parameters. Although many experiments of Be diffusion in InGaAs are reported in literature, they are done under different experimental conditions. Therefore, in the following, we consider only experiments where the only difference in conditions are annealing temperatures and durations. ${ }^{2,6,10,16}$ Specifically, we consider experiments where: (i) the doping process is done by gas source molecular beam epitaxy rather than ion implantation or in-diffusion from an extended source, both of which will induce implantation damage or surface effect, which are difficult to account for in a simulation; (ii) the gas source molecular beam epitaxy parameters are similar. It is well known that such parameters will strongly influence the material's transport properties.

We first assumed the following trial diffusion mechanism:

$$
\mathrm{Be}_{\mathrm{III}}^{++}+\mathrm{III}_{\mathrm{i}}^{0} \leftrightharpoons \mathrm{Be}_{\mathrm{i}}^{++}
$$

where $\mathrm{Be}_{\text {III }}^{++}$denotes a doubly positive $\mathrm{Be}$ atom in a group-III sublattice position, $\mathrm{III}_{\mathrm{i}}^{0}$ represents a neutral $\mathrm{Ga}$ or In interstitial, and $\mathrm{Be}_{i}^{++}$is a doubly positive $\mathrm{Be}$ atom in an interstitial position. The charge states are selected based on DFT estimates of state occupancies at experimental temperatures (see section III C). The corresponding diffusion equations are the following:

$$
\begin{aligned}
\frac{\partial C_{i}}{\partial t} & =\frac{\partial}{\partial t}\left(D_{i} \frac{\partial C_{i}}{\partial x}-n D_{i} \frac{C_{i}}{p} \frac{\partial p}{\partial x}\right)-\frac{\partial C_{s}}{\partial t} \\
\frac{\partial C_{I}}{\partial t} & =\frac{\partial}{\partial t}\left(D_{I} \frac{\partial C_{I}}{\partial x}-r D_{I} \frac{C_{I}}{p} \frac{\partial p}{\partial x}\right)+\frac{\partial C_{s}}{\partial t}
\end{aligned}
$$

where $C_{i}$ is the concentration of $\mathrm{Be}_{\mathrm{i}}^{n+}$ where $n=2, C_{I}$ is the concentration of $\mathrm{I}_{\mathrm{III}}^{r+}$ where $r=0, C_{s}$ is the concentration of $\mathrm{Be}_{\mathrm{III}}, D_{i}$ and $D_{I}$ are the diffusivities of $\mathrm{Be}_{\mathrm{i}}$ and $\mathrm{III}_{\mathrm{i}}^{r+} ; p$ is the hole concentration; $n$ and $r$ are Be interstitial and III-group interstitial charge numbers respectively. The best fit achieved with Eq. 5 at several temperatures is shown in Fig. 12. We note that this model fit the data reasonably well at $700{ }^{\circ} \mathrm{C}, 750^{\circ} \mathrm{C}$, $800^{\circ} \mathrm{C}$, but shows a serious discrepancy at $900^{\circ} \mathrm{C}$.

Therefore we tried another diffusion mechanism:

$$
\mathrm{Be}_{\mathrm{III}}^{++}+\mathrm{III}_{\mathrm{i}}^{-} \rightleftharpoons \mathrm{Be}_{\mathrm{i}}^{+}
$$

where $\mathrm{III}_{\mathrm{i}}^{-}$represents a singly negative Ga or In interstitial and $\mathrm{Be}_{\mathrm{i}}^{+}$is a singly positive Be atom in an interstitial position. The corresponding partial differential equations are similar to Eq. 6 but with $n=1$ and $r=-1$. The

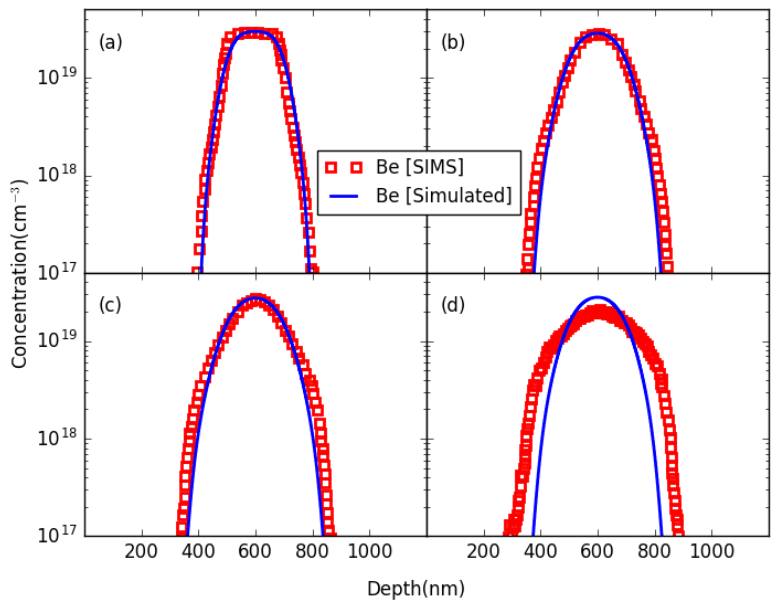

Fig. 12: Experimental and simulated Be profiles for the mechanism of Eq. 5 obtained with the continuum method under different annealing conditions: (a)180 s at $700{ }^{\circ} \mathrm{C}$, (b) $120 \mathrm{~s}$ at $750{ }^{\circ} \mathrm{C},(\mathrm{c}) 60 \mathrm{~s}$ at $800^{\circ} \mathrm{C}$, (d) $10 \mathrm{~s}$ at $900^{\circ} \mathrm{C}{ }^{2,10,16}$

best fit achieved by Eq. 7 for different temperatures is shown in Fig. 13. We see that it agrees with the data at $900^{\circ} \mathrm{C}$, but does not fit well at $700{ }^{\circ} \mathrm{C}, 750{ }^{\circ} \mathrm{C}, 800^{\circ} \mathrm{C}$, especially in the high concentration region. This is the opposite of Eq. 5. Since we have already shown based on DFT calculations that at low temperatures, $\mathrm{Ga}_{\mathrm{i}}^{0}$ population dominates, and $\mathrm{Ga}_{\mathrm{i}}^{-}$will only have a significant population at the highest temperatures among considered here, that the models assuming a single $\mathrm{Ga}_{\mathrm{i}}$ charge state only fit the experimental data in different temperature ranges is consistent with DFT results. This suggests that we should include at least two mechanisms with different temperature dependences, one dominating at lower temperatures, another at higher temperatures. The integrated model proposed is:

$$
\begin{array}{rr}
\mathrm{Be}_{\mathrm{III}}^{++}+\mathrm{III}_{\mathrm{i}}^{0} \rightleftharpoons & \mathrm{Be}_{\mathrm{i}}^{++}, \\
\mathbb{\Downarrow} & \mathbb{\Downarrow} \\
\mathrm{Be}_{\mathrm{III}}^{++}+\mathrm{III}_{\mathrm{i}}^{-} \rightleftharpoons & \mathrm{Be}_{\mathrm{i}}^{+} .
\end{array}
$$

The horizontal reactions are elementary diffusion reactions, while the vertical arrows indicate changes in charge state. Such changes are due to changes in electronic state occupancies and are much faster than diffusion reactions; therefore, we can assume that the equilibrium between different charge states is instantaneous and this equilibrium is determined by local Fermi level and transition energy. The results of fits using this model are shown in Fig. 14. Clearly, the model performs very well over the entire temperature range. 


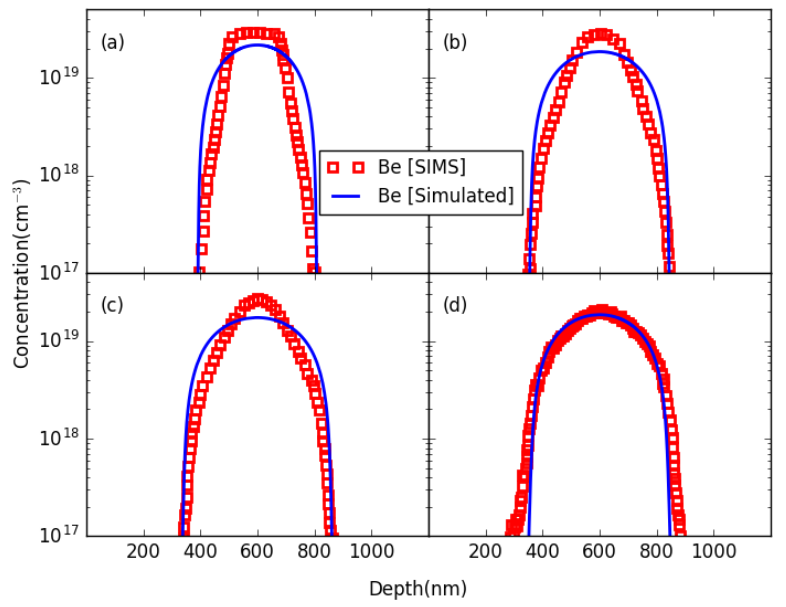

Fig. 13: Experimental and simulated Be profiles for the mechanism of Eq. 7 obtained with the continuum method under different annealing conditions: (a)180 s at $700^{\circ} \mathrm{C},(\mathrm{b}) 120 \mathrm{~s}$ at $750{ }^{\circ} \mathrm{C},(\mathrm{c}) 60 \mathrm{~s}$ at $800^{\circ} \mathrm{C}$ (d) $10 \mathrm{~s}$ at $900^{\circ} \mathrm{C}^{2,10,16}$

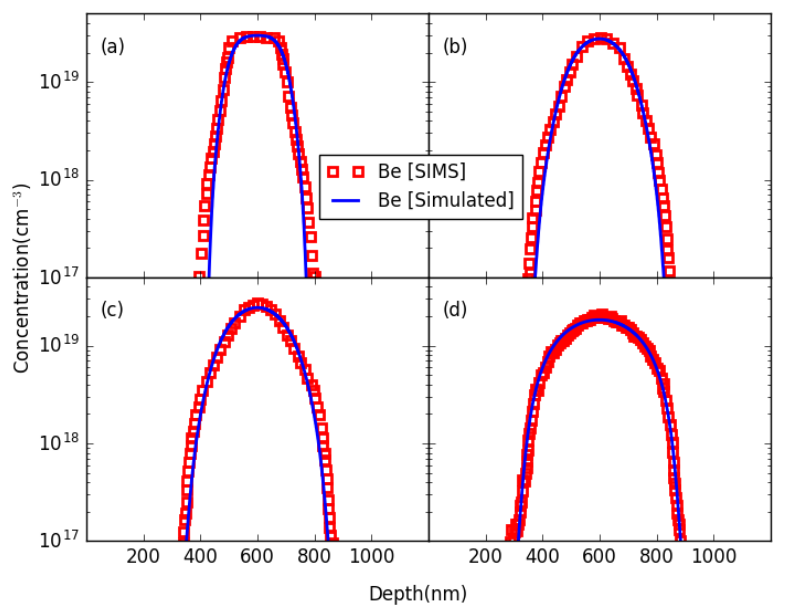

Fig. 14: Experimental and simulated Be profiles for the mechanism of Eq. 8 obtained with the continuum method under different annealing conditions: (a)180 s at $700^{\circ} \mathrm{C}$, (b) $120 \mathrm{~s}$ at $750^{\circ} \mathrm{C},(\mathrm{c}) 60 \mathrm{~s}$ at $800^{\circ} \mathrm{C}$, (d) $10 \mathrm{~s}$ at $900^{\circ} \mathrm{C} .{ }^{2,10,16}$

\section{E. Kinetic Monte Carlo simulations}

Although the continuum treatment can provide a good fit to experimental data, its application is limited to simple diffusion mechanisms. The reason is that the number of differential equations will grow as the diffusion mechanism becomes more and more complex, the differential equations need to be coupled and advanced numerical methods need to be used. Compared with the continuum treatment, kinetic Monte Carlo (KMC) simulation is

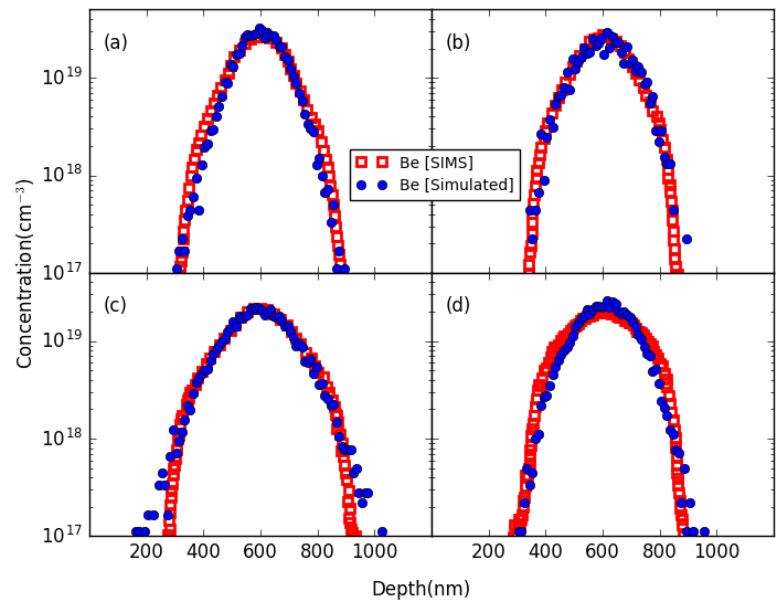

Fig. 15: Experimental and simulated Be profiles for mechanism Eq. 9 by kinetic Monte Carlo method under different annealing conditions: (a) $240 \mathrm{~s}$ at $750^{\circ} \mathrm{C}$, (b) $60 \mathrm{~s}$ at $800^{\circ} \mathrm{C},(\mathrm{c}) 180 \mathrm{~s}$ at $800^{\circ} \mathrm{C},(\mathrm{d}) 10 \mathrm{~s}$ at $900^{\circ} \mathrm{C} .{ }^{2,10,16}$

very suitable for simulating very complex diffusion mechanisms because one simply needs to add reactions into an event list, and the algorithm will handle complicated correlations implicitly.

Based on the parameters derived from the continuum model, we also reproduced the experiments of grown-in Be diffusion in InGaAs by KMC simulations. Here, we assume that Be diffusion in InGaAs can be described by the following kick-out mechanism:

$$
\begin{array}{cr}
\mathrm{Be}_{\mathrm{III}}^{++}+\mathrm{III}_{\mathrm{i}}^{0} \rightleftharpoons & \mathrm{Be}_{\mathrm{i}}^{++}, \\
\mathrm{Be}_{\mathrm{III}}^{++}+\mathrm{III}_{\mathrm{i}}^{-} \rightleftharpoons & \mathrm{Be}_{\mathrm{i}}^{+}, \\
& \sqrt{\mathbb{1}} \\
& \mathrm{Be}_{\mathrm{i}}^{0},
\end{array}
$$

where $\mathrm{Be}_{\mathrm{i}}^{0}$ denotes a neutral Be interstitial. Using parameters show in Table IV, we successfully fit experiment data as shown in Fig. 15.

We notice in Table IV that while the charge states in our KMC simulations are consistent with the DFT results, the migration energies are quite different. This is not surprising since we had to adopt several simplifications in the KMC simulation imposed by the program; specifically, the simulation neglected reactions involving As and treated $\mathrm{Ga}$ and In as the same object. In principle, Ga and In need to be treated differently in the KMC simulations because their reaction parameters are different. Also, reactions involving As have sufficiently small reaction energies and barriers to contribute substantially to the process (see Fig. 5 and Table II). Therefore, the KMC parameters are not directly comparable with the DFT results and one needs instead to adopt a more advanced $\mathrm{KMC}$ simulation in the future. The migration 
TABLE IV: Atomistic parameters of the atom species involved in Be diffusion used in the MMonCa. $D_{m, 0}$ is the diffusivity prefactor, $E_{m}$ the migration energy and $e_{t}$ the transition level measured from the valence band edge. Prefactors for equilibrium concentrations and formation energies are $[X]_{0}^{e q}$ and $E_{F}$. Parameters left blank will be calculated by MMonCa using Eq. 1 and Eq. 3.

\begin{tabular}{|c|c|c|c|c|c|c|}
\hline & $\mathrm{Be}_{\mathrm{III}}^{++}$ & $\mathrm{III}_{\mathrm{i}}^{0}$ & $\mathrm{III}_{\mathrm{i}}^{-}$ & $\mathrm{Be}_{i}^{0}$ & $\mathrm{Be}_{\mathrm{i}}^{+}$ & $\mathrm{Be}_{\mathrm{i}}^{++}$ \\
\hline$D_{m, 0} \times\left(\mathrm{cm}^{2} / \mathrm{s}\right)$ & 0 & $8.40 \times 10^{-3}$ & $8.40 \times 10^{-3}$ & 0 & $5.34 \times 10^{3}$ & $6.71 \times 10^{-3}$ \\
\hline$E_{m}(e V)$ & 5 & 1.44 & 1.44 & 5 & 3.09 & 1.41 \\
\hline$e_{t}(T=0)(\mathrm{eV})$ & & & 0.55 & & 1.00 & 0.80 \\
\hline$[X]_{0}^{e q}\left(\times 10^{25} \mathrm{~cm}^{-3}\right)$ & 1 & $5.00 \times 10^{25}$ & & $1.33 \times 10^{4}$ & & \\
\hline$E_{F}(e V)$ & 0 & 2.13 & & 1.48 & & \\
\hline
\end{tabular}

energies of $\mathrm{Be}_{\mathrm{i}}^{+}$and $\mathrm{Be}_{\mathrm{i}}^{++}$are quite different, which is consistent with the continuum method.

\section{SUMMARY}

In this work, a physically motivated multiscale modeling of grow-in Be diffusion in InGaAs has been presented. In order to evaluate the importance of different diffusion mechanisms, we investigated the reaction energies and elementary diffusion processes in Be-doped $\operatorname{In}_{0.5} \mathrm{Ga}_{0.5} \mathrm{As}$ using DFT. The energy barrier for Frank-Turnbull mechanism was found to be much higher than for the kick-out mechanism, therefore, the kick-out mechanism is likely to be primarily responsible for Be diffusion in the experimental temperature range. In contrast to previous models, the roles of $\mathrm{Ga}$ and $\mathrm{In}$ are found to be different in the kick-out reactions, specifically, kicking out of Ga is an exothermic reaction while kicking out of In is endothermic. Therefore, accurate simulations should treat Ga and In separately. The reaction energy for kicking out of As is comparable to that for kicking out of $\mathrm{Ga}$ and In, thus one should revisit the commonly accepted assumption that As is not involved in Be diffusion. Besides providing a mechanistic insight, the ab initio simulations also provided physically motivated parameters which served as input for the continuum and KMC simulations. Continuum modeling indicated that different charge states of $\mathrm{Ga}$ and Be interstitials are contributing to the diffusion mechanism at different temperatures and both continuum and $\mathrm{KMC}$ simulations agree well with experimental results. The differences in optimal KMC parameters and DFT suggested parameters points to the deficiency of common approximate treatments of $\mathrm{Ga}$, In and As.

\section{ACKNOWLEDGEMENT}

We acknowledge industry financial support provided by GlobalFoundries, and a research scholarship provided by the Energy Research Institute at Nanyang Technological University (ERIAN). *mpemanzh@nus.edu.sg

† nacho@synopsys.com

1 J. a. del Alamo, Nature 479, 317 (2011).

2 J. Marcon, M. Ihaddadene, and K. Ketata, Journal of Crystal Growth 253, 174 (2003).

3 J. C. Hu, M. D. Deal, and J. D. Plummer, Journal of Applied Physics 78, 1595 (1995).

4 S. Koumetz, J. Marcon, K. Ketata, M. Ketata, C. DubonChevallier, P. Launay, and J. L. Benchimol, Applied Physics Letters 67, 2161 (1995).

5 J. Marcon, S. Koumetz, K. Ketata, M. Ketata, and J. G. Caputo, The European Physical Journal Applied Physics 8, 7 (1999).

6 S. D. Koumetz, P. Martin, and H. Murray, Journal of Applied Physics 116, 103701 (2014).

7 S. Koumetz, J. Marcon, K. Ketata, M. Ketata, F. Lefebvre, P. Martin, and P. Launay, Materials Science and Engineering B 37, 208 (1996).

8 J. Marcon, S. Gautier, K. Koumetz, K. Ketata, M. Ketata, and P. Launay, Solid State Communications 101, 159 (1997).
9 J. Marcon, S. Gautier, S. Koumetz, K. Ketata, and M. Ketata, Computational Materials Science 10, 28 (1998).

${ }^{10}$ K. Ketata, M. Ketata, S. Koumetz, and J. Marcon, Europhysics Letters (EPL) 45, 348 (1999).

11 K. Ketata, M. Ketata, S. Koumetz, J. Marcon, and O. Valet, Physica B: Condensed Matter 273-274, 823 (1999).

12 S. Koumetz, O. Valet, J. Marcon, and M. Ketata, Materials Science and Engineering B 71, 171 (2000).

13 S. Koumetz, M. Ketata, K. Ketata, M. Ihaddadene, E. Joubert, and C. Dubois, Journal of Crystal Growth 220, 46 (2000).

14 M. Ihaddadene, S. Koumetz, O. Latry, K. Ketata, M. Ketata, and C. Dubois, Materials Science and Engineering B 80, 73 (2001).

15 M. Ihaddadene, J. Marcon, M. Idrissi-Benzohra, K. Ketata, S. Demichel, J. Flicstein, J. L. Pelouard, and M. Ketata, Computational Materials Science 24, 257 (2002).

16 S. Koumetz and C. Dubois, Journal of Crystal Growth 252, 14 (2003). 
17 F. Legrain and S. Manzhos, Journal of Power Sources 274, 65 (2015).

18 I. Martin-Bragado, A. Rivera, G. Valles, J. L. GomezSelles, and M. J. Caturla, Computer Physics Communications 184, 2703 (2013).

19 W. Koch and M. C. Holthausen, A Chemist's Guide to Density Functional Theory (Wiley-VCH Verlag GmbH, Weinheim, FRG, 2001).

${ }^{20}$ R. G. Parr, in Horizons of Quantum Chemistry, Vol. 47 (Springer Netherlands, Dordrecht, 1980) pp. 5-15.

21 J. P. Perdew, K. Burke, and M. Ernzerhof, Phys. Rev. Lett. 77, 3865 (1996), arXiv:0927-0256(96)00008 [10.1016].

${ }^{22}$ G. Kresse and J. Hafner, Phys. Rev. B 48, 13115 (1993).

${ }^{23}$ G. Kresse and J. Furthmüller, Comput. Mat. Sci. 6, 15 (1996).

${ }^{24}$ G. Kresse and J. Furthmüller, Physical Review B 54, 11169 (1996).

25 P. E. Blochl, Physical Review B 50, 17953 (1994), arXiv:arXiv:1408.4701v2.

${ }^{26}$ G. Kresse, Physical Review B 59, 1758 (1999).
27 J. D. Pack and H. J. Monkhorst, Physical Review B 16 , 1748 (1977).

${ }^{28}$ G. Henkelman and H. Jonsson, The Journal of Chemical Physics 113, 9978 (2000).

${ }^{29}$ G. Henkelman, B. P. Uberuaga, and H. Jonsson, The Journal of Chemical Physics 113, 9901 (2000).

30 H.-P. Komsa and A. Pasquarello, Physica B: Condensed Matter 407, 2833 (2012).

31 H.-P. Komsa, A. Pasquarello, C. De Simulatio, and E. Atomique, J. Phys.: Condens. Matter 24, 45801 (2012).

32 W. Tang, E. Sanville, and G. Henkelman, Journal of Physics: Condensed Matter 21, 084204 (2009).

33 K. Momma and F. Izumi, Journal of Applied Crystallography $44,1272(2011)$.

34 L. T. Ho, Applied Physics Letters 35, 409 (1979).

${ }^{35}$ F. Legrain and S. Manzhos, "Comparison of lithium, sodium, and magnesium storage in pure and gallium-doped germanium: a first-principles study," (2016), (unpublished). 